\section{Upfront combination therapy: does the AMBITION study herald a new era in the treatment of pulmonary arterial hypertension?}

\author{
Steven D Nathan, ${ }^{1}$ Paul A Corris ${ }^{2}$
}

A new treatment paradigm for pulmonary arterial hypertension (PAH) has emerged, heralded by the recently published results of the AMBITION study in a recent issue of the New England Journal of Medicine. ${ }^{1}$ While the practice of combination therapy is not new, this has previously been implemented in a sequential fashion. The AMBITION study is the first study comparing the use of upfront combination therapy in patients with treatment-naïve incident PAH who were randomised to therapy with ambrisentan plus tadalafil versus ambrisentan or tadalafil monotherapy. The results were initially presented at the European Respiratory Society meeting in September 2014 with the primary publication in the New England Journal of Medicine in August 2015. ${ }^{1}$

The primary efficacy endpoint of the AMBITION study was time to first clinical failure (TTCF) defined as either death, hospitalisation for worsening $\mathrm{PAH}$, disease progression $(>15 \%$ decline in 6 min walk test (MWT) from baseline with WHO functional class III or IV symptoms) or unsatisfactory clinical response (WHO functional class III symptoms while in the study for at least 6 months with a decrease in 6 MWT distance from baseline). The latter three events were all adjudicated and multiple secondary endpoints, including change in the 6 min walk distance, were also evaluated. There were 610 patients enrolled in the study of whom 500 constituted the primary analysis set (PAS). ${ }^{1}$ The other 105 patients were excluded from the PAS after a protocol amendment aimed at excluding those patients with risk factors for left ventricular diastolic dysfunction. Patients were randomised in a $2: 1: 1$

\footnotetext{
${ }^{1}$ Advanced Lung Disease and Transplant Program, Department of Medicine, Inova Heart and Vascular Institute, Falls Church, Virginia, USA; ${ }^{2}$ Institute of Cellular Medicine, Newcastle University and The Newcastle upon Tyne Hospitals NHS Foundation Trust, Newcastle upon Tyne, UK

Correspondence to Dr Steven D Nathan, Advanced Lung Disease and Transplant Program, Department of Medicine, Inova Heart and Vascular Institute, Falls Church VA 22042, USA; steven.nathan@inova.org
}

fashion to upfront combination therapy or monotherapy with ambrisentan or tadalafil. The study demonstrated unequivocally that upfront combination therapy was indeed superior to monotherapy with either single agent based on the selected end points of the study. The HR for TTCF for combination therapy compared with the pooled monotherapy arms was 0.50 (CI 0.35 to $0.72, \mathrm{p}<0.001$ ). In numerical terms, approximately $89 \%$ of the combination therapy patients were event-free at 1 year versus $75.5 \%$ for the pooled monotherapy arms (based on Kaplan-Meier estimates). At 2 years, the difference was $79.7 \%$ vs $63.2 \%$, while at 3 years, it was $68 \%$ vs $56 \%$. This difference persisted across all subgroups analysed. Two notable secondary endpoints supported the primary efficacy analysis. Specifically, the NT pro-BNP was significantly reduced for the combination therapy arm compared with the ambrisen$\tan (\mathrm{p}=0.01)$ and tadalafil $(\mathrm{p}<0.001)$ arms. Change in the $6 \mathrm{MWT}$ distance at 24 weeks was also significantly improved for the combination arm $(+49 \mathrm{~m})$ compared with both the ambrisentan $(+27 \mathrm{~m}$, $\mathrm{p}=0.001)$ and tadalafil monotherapy arms $(+22.7 \mathrm{~m}, \mathrm{p}=0.003)$.

This study has answered an important question, but has also raised many more areas for debate: (1) Is upfront combination therapy now the standard for all patients with newly diagnosed PAH? (2) Are there still patients that should be treated with monotherapy and what then of the role of sequential therapy? (3) Is it truly the synergistic action of the two agents that are causing the difference, or are we simply doubling the chances of individual patients being on a medication that is best suited to them? This conundrum does not impact the practical application of the AMBITION findings since we cannot predict how individual patients will respond to specific drugs. (4) Is the concept of upfront combination therapy applicable to any endothelin receptor antagonist (ERA) and phosphodiesterase (PDE) 5 inhibitor (or direct soluble guanylate stimulators) combination or is it specific to the two agents used in this study? (5) What of the cost and will payers assume this financial burden? (6) What are the implications for future PAH clinical trial design? (7) How will the AMBITION results shape future guideline recommendations?

The question as to whether or not the results of this study are drug-specific or if any combination of ERA and PDE5 would result in the same benefit is extremely important to address, especially with the potential availability of cheaper within class generics. The original PDE5 inhibitor, sildenafil, is currently available as a generic, while bosentan (the first ERA) is soon to come off patent. Although drugs within the same class have the same general mechanism of action, each has its own nuanced unique activity characteristics. $^{2} 3$ Specifically, in addition to their different pharmacokinetic profiles, sildenafil and tadalafil have different affinities for the various PDE subgroups, while the ERAs have different affinities for the endothelin A and B receptors. Indeed, dual receptor blockade versus endothelin A specific blockade has been an issue of ongoing debate. ${ }^{3}$ There is also the issue of drug-drug interactions (DDIs) which might affect the pharmacokinetic profile of both agents. This has implications for side effects and efficacy with a spectrum of possible outcomes that include synergism, additive affects and possibly even antagonism. Therefore, the results of the AMBITION trial cannot be generalised to other agents yet. This is reinforced by the results of two negative studies using bosentan and sildenafil in combination. The COMPASS 2 study was perhaps the longest running trial in $\mathrm{PAH}$ and took $>7$ years to complete. ${ }^{4}$ This study evaluated the effects of add-on bosentan to patients who were on background sildenafil. It enrolled 334 patients, but failed to meet its primary endpoint of time to first morbidity or mortality. The corollary study to this evaluated sildenafil as add-on therapy to bosentan. This negative study is yet to be presented or published, but the results are available online within the clinicaltrials.gov website. ${ }^{5}$ It is conceivable that these studies were negative due to differences in the respective trial designs. Arguably, it was the DDI profiles of these two specific agents that resulted in negative trials, specifically bosentan has been shown to reduce sildenafil exposure, while sildenafil has been demonstrated to increase the bosentan area under the curve. ${ }^{6}$ It is noteworthy that both trials did not evaluate upfront combination treatment, but rather 
sequential add-on therapy. Nonetheless, it is difficult to postulate that the results would have been any different if both drugs were administered upfront together as per the AMBITION study design. On the other hand, it remains unknown how other ERA plus PDE5 inhibitors (or soluble guanylate cyclase activators) combinations might perform and these need to be subjected to their own study in order to demonstrate efficacy. In recognition of the AMBITION data, the recent European Society of Cardiology and European Respiratory Society guidelines published in October 2015 afford initial drug combination therapy with ambrisentan and tadalafil, the only class I recommendation (with level B evidence) for patients with functional class II and III PAH. ${ }^{7}$

Do the results of AMBITION herald a new era where upfront combination therapy with these agents becomes the standard for all patients with PAH? It is well established that there are subgroups of patients who do very well long-term on monotherapy. These subgroups are somewhat small with reported ranges of 31\%$56 \%$ in long-term open-label extension trials. $^{8-12}$

Unfortunately, we are not very good at identifying these patients a priori to be confident that monotherapy will suffice. It is also well recognised that it can be difficult to rescue patients with $\mathrm{PAH}$ who deteriorate. This, therefore, begs the question of why wait for deterioration and deny upfront combination to all patients? On the other end of the severity spectrum, there is the subgroup of patients who present with advanced symptoms who require parenteral prostanoid therapy as their first-line treatment. Whether these patients should be treated with parenteral therapy plus oral therapy (or therapies) upfront poses a different question. This has been answered to an extent in other clinical trials, most notably the PACES trial. ${ }^{13}$ PACES was a positive study of add-on sildenafil to patients who were already on intravenous epoprostenol. Whether clinicians extrapolate from the AMBITION dataset that oral therapy with ambrisentan plus tadalafil can substitute for sildenafil, while seemingly pragmatic and intuitive, requires its own study for further validation.

Upfront combination therapy for $\mathrm{PAH}$ is an expensive undertaking both at the individual patient and societal levels. How will this new treatment paradigm be managed by individual and national payers? There is indeed a cogent pharmacoeconomic argument for upfront combination therapy which is embedded within the primary composite TTCF endpoint. Examination of the Kaplan-Meier curve is noteworthy that one of the biggest step-downs in the monotherapy arm occurs at 6 months, which marks the first time that patients could meet the failure to improve component of the composite. Interesting, however, is that many of these events were actually hospitalisations, thereby underscoring the favourable cost-benefit of upfront combination therapy.

Lastly, how will the results of AMBITION influence the implementation and design of future PAH clinical trials. It is largely accepted that placebo trials are unethical and all clinical trial designs now allow patients to be on at least one PAH therapy. If indeed, the combination of ambrisentan plus tadalafil is the new standard, then arguably it becomes unethical to have patients on anything short of this in the context of future clinical trials. If this sets the new standard of care, then future trials will need to have this as the comparator group. An AMBITION-like study design and endpoint will result in an event rate that is very low and will require a large patient cohort followed over a long period of time to show a difference. What of the cost to do such a trial, both in terms of financial burden and the human cost of committing many patients with $\mathrm{PAH}$ to a lengthy trial which may or may not be positive. Do we need to move back to the era of smaller, shorter duration studies? While this might fly in the face of the current viewpoint of larger and longer equates to more robust data, this might be a pragmatic necessity. Such studies will require modification of our endpoints for an achievable event rate. Prior composites of time to clinical worsening could be regarded as a defensive strategy where as long as patients stayed stable or even had some mild deterioration, they did not breach any component of the endpoint. The time to clinical failure endpoint raised the bar in that 'clinical failure' included a component that required some demonstrable benefit, however small, at 6 months. In order to power future shorter-term studies, the bar needs to be raised further and the time to clinical failure definition modified with expectations of a greater improvement. This might include the old standard and enduring $6 \mathrm{MWT}$ with perhaps the expectation of a $10 \%$ or $15 \%$ increase in the distance. It could also include a threshold for haemodynamic improvement, evidence of right ventricular regression or biomarker (pro-NT
BNP) concordance. Perhaps time to clinical improvement becomes the new endpoint, with time to clinical worsening an important secondary endpoint. An alternate approach might be to combine these two disparate outcomes in a competing risk analysis over a medium-term (eg, 612 months) period with three possible endpoints of worsening, stability or improvement.

The field of PAH has come a long way in recent years and clinicians are now faced with many different choices. The AMBITION study answers an important question and may change clinical practice meaningfully. However, it is ultimately up to the individual clinician how they apply this new information in the context of other choices in their day-to-day practice, since each patient is nuanced and invariably do not fulfil the exact criteria of subjects enrolled into clinical trials. We still have much to learn and a long way to go with ample room and need for future therapies. The real challenge is to be able to predict the response a patient will show to each class of drug and use this to inform decision-making. Hopefully, AMBITION will serve to encourage further novel drug development, trials of treatment strategies and future pragmatic innovative clinical trial designs.

Competing interests SDN has consulted for, is on the speaker's bureau and received research funding from the sponsor of the AMBITION study, Gilead Pharmaceuticals. PAC has received research grant funding from Bayer and Actelion and sat on remunerated advisory boards for Bayer, Actelion and GSK.

Provenance and peer review Not commissioned; externally peer reviewed.

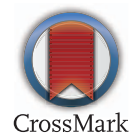

To cite Nathan SD, Corris PA. Thorax 2016;71:107109.

Received 17 September 2015

Revised 19 October 2015

Accepted 27 October 2015

Published Online First 23 November 2015

Thorax 2016;71:107-109.

doi:10.1136/thoraxjnl-2015-207854

\section{REFERENCES}

1 Galiè N, Barberà JA, Frost AE, et al. Initial use of ambrisentan plus tadalafil in pulmonary arterial hypertension. N Engl J Med 2015;373:834-44.

2 Ghofrani HA, Voswinckel $R$, Reichenberger $F$, et al. Differences in hemodynamic and oxygenation responses to three different phosphodiesterase-5 inhibitors in patients with pulmonary arterial hypertension: a randomized prospective study. J Am Coll Cardiol 2004;44:1488-96.

3 Opitz CF, Ewert R, Kirch W, et al. Inhibition of endothelin receptors in the treatment of pulmonary 
arterial hypertension: does selectivity matter? Eur Heart J 2008;16:1936-48.

4 McLaughlin V, Channick R, Ghofrani HA, et al. Bosentan added to sildenafil therapy in patients with pulmonary arterial hypertension. Eur Respir J 2015;46:405-13.

5 Assess the efficacy and safety of sildenafil when added to bosentan in the treatment of pulmonary arterial hypertension. ClinicalTrials.gov Identifier: NCT00323297. https://clinicaltrials.gov/ct2/show/ results/NCT00323297?term=sildenafil+and +bosentan\&sect $=$ X01256\#all

6 Paul GA, Gibbs ISR, Boobis AR, et al. Bosentan decreases the plasma concentration of sildenafil when coprescribed in pulmonary hypertension. Br J Clin Pharmacol 2005;60: 107-12.
7 Galiè N, Humbert M, Vachiery JL, et al. 2015 ESC/ERS guidelines for the diagnosis and treatment of pulmonary hypertension: the Joint Task Force for the diagnosis and treatment of pulmonary hypertension of the European Society of Cardiology (ESC) and the European Respiratory Society (ERS): endorsed by: Association for European Paediatric and Congenital Cardiology (AEPC), International Society for Heart and Lung Transplantation (ISHLT). Eur Respir J 2015;46: 903-75.

8 Sitbon O, Badesch DB, Channick RN, et al. Effects of the dual endothelin receptor antagonist bosentan in patients with pulmonary arterial hypertension: a 1-year follow-up study. Chest 2003;124: 247-54.

9 Simonneau G, Galiè N, Jansa P, et al. Long-term results from the EARLY study of bosentan in WHO functional class II pulmonary arterial hypertension patients. Int J Cardiol 2014;172:332-9.

10 Oudiz RJ, Galiè N, Olschewski H, et al. Long-term ambrisentan therapy for the treatment of pulmonary arterial hypertension. J Am Coll Cardiol 2009:54:1971-81.

11 Rubin LJ, Badesch DB, Fleming TR, et al. Long-term treatment with sildenafil citrate in pulmonary arterial hypertension: the SUPER-2 study. Chest 2011:140:1274-83.

12 Oudiz RJ, Brundage $\mathrm{BH}$, Galiè $\mathrm{N}$, et al. Tadalafil for the treatment of pulmonary arterial hypertension. J Am Coll Cardiol 2012:60:768-74.

13 Simonneau G, Rubin LJ, Galie N, et al. Addition of sildenafil to long-term intravenous epoprostenol therapy in patients with pulmonary arterial hypertension. Ann Intern Med 2008;149:521-30. 